\title{
gs \\ Fast readout of a single Cooper-pair box using its quantum capacitance
}

\author{
F. Persson, ${ }^{*}$ C. M. Wilson, M. Sandberg, and P. Delsing ${ }^{\dagger}$ \\ Department of Microtechnology and Nanoscience (MC2), Chalmers University of Technology, SE-412 96 Göteborg, Sweden
}

(Received 26 March 2010; published 27 October 2010)

\begin{abstract}
We have fabricated a single Cooper-pair box (SCB) together with an on-chip lumped element resonator. By utilizing the quantum capacitance of the SCB, its state can be read out by detecting the phase of a radiofrequency signal reflected off the resonator. The resonator was optimized for fast readout. By studying quasiparticle tunneling events in the SCB, we have characterized the performance of the readout and found that we can perform a single-shot parity measurement, with a signal-to-noise ratio of 1 , in approximately $50 \mathrm{~ns}$. This is an order of magnitude faster than previously reported measurements.
\end{abstract}

DOI: 10.1103/PhysRevB.82.134533

PACS number(s): 85.25.Cp, 73.23.Hk, 42.50.Dv

\section{INTRODUCTION}

Superconducting devices based on Josephson junctions have successfully been used in many different kinds of applications, including very sensitive magnetometers based on superconducting quantum interference devices (SQUIDs), bolometric detectors, mixers, and parametric amplifiers. They have also been suggested to be strong candidates as building blocks for a quantum computer. ${ }^{1-4}$ They are easily fabricated with standard lithographic techniques and can be integrated with other electrical circuits. This gives them the potential to be scalable. Devices made with very small tunnel junctions can exploit charging effects. Such Coulombblockade devices are also widely used in measurements, for example, the single-electron transistor (SET). ${ }^{5}$ The radiofrequency (rf) version of the SET is the worlds most sensitive electrometer. ${ }^{6,7}$

The single Cooper-pair box (SCB) (Refs. 8-10) is one of the simplest Coulomb-blockade devices, involving a single Josephson junction. The SCB is very sensitive to the presence of quasiparticles which suggested its use as a potential radiation detector. ${ }^{11}$ The presence of quasiparticles can be measured by detecting the charge on the SCB island using an external SET. ${ }^{12}$ In this paper, we characterize an intrinsic method for reading out the SCB which relies on the curvature of its energy bands. This method is both faster and is predicted to have less backaction than using an SET. The curvature of the energy bands of the SCB (with respect to gate charge) gives rise to the so-called quantum capacitance ${ }^{13,14}$ and has been utilized in a number of experiments, for example, in measurements of longitudinal dressed states of a driven SCB. ${ }^{15,16}$ It has also been used to study the ground state of two coupled qubits ${ }^{17}$ and to study quasiparticle poisoning of a SCB qubit. ${ }^{18}$ In Ref. 18, they used a resonator with a bandwidth of $200 \mathrm{kHz}$ which limited the speed the their measurements. Here we use the random tunneling of quasiparticles to characterize the performance of the quantum capacitance readout. We show that we can perform a parity measurement of the SCB an order of magnitude faster than has previously been reported, including measurements using rf-SETs. We also show that we can prepare the SCB in a certain parity state with a high probability.

This paper is structured as follows: in Sec. II, we present the theory behind the readout technique of using the quantum capacitance. In Sec. III, we show how the samples were designed and fabricated and then in Sec. IV we present the measurements done to characterize the readout.

\section{THEORY}

\section{A. Cooper-pair box}

The sample under investigation is a SCB and is shown in Figs. 1(a) and 1(b). The SCB consists of a superconducting island connected to a large reservoir by a Josephson junction. The Josephson junction is made in a SQUID configuration to allow the Josephson energy, $E_{J}$, to be tuned by applying a magnetic field through the loop. The SCB is also characterized by the electrostatic energy, $E_{e l}=E_{Q}\left(n-n_{g}\right)^{2}$, which is the energy required to add an extra Cooper pair to the island. Here, $n$ is the number of Cooper pairs that have tunneled onto the island and $n_{g}=C_{g} V_{g} / 2 e$ is the normalized gate voltage, where $C_{g}$ is the capacitance between the gate electrode and the island. If the capacitance of the island, $C_{\Sigma}$, is small enough, the Cooper-pair charging energy, $E_{Q}=(2 e)^{2} / 2 C_{\Sigma}$, will dominate over the Josephson energy, $E_{J}$, and the temperature, $k_{B} T$. In this case, the charge fluctuation on the island will be small. The number of excess Cooper pairs on the island, $n$, is then a good quantum number and the charge of the island can be well controlled by the external gate voltage, $V_{g}$. For $E_{J} \ll E_{Q}$ and $0<n_{g}<1$, only two charge states will be of interest: $|0\rangle$ and $|1\rangle$, corresponding to zero $(n=0)$ or one $(n=1)$ extra Cooper pair on the island. Then the truncated Hamiltonian of the Cooper-pair box can be written as

$$
H=-\frac{1}{2} E_{Q}\left(1-2 n_{g}\right) \sigma_{z}-\frac{1}{2} E_{J} \sigma_{x}
$$

where $\sigma_{x}, \sigma_{z}$ are the Pauli spin matrices. Here we have ignored all state-independent terms of the Hamiltonian. The two eigenenergies for this Hamiltonian are plotted in Fig. 1(d) as a function of $n_{g}$. At the degeneracy point $n_{g}=0.5$, where the electrostatic energies of the two charge states cross, we get an avoided level crossing with a splitting between the ground and excited state equal to $E_{J}$. In the same graph, we have also plotted the expectation value of the island charge $\langle n\rangle$ for each energy eigenstate. 

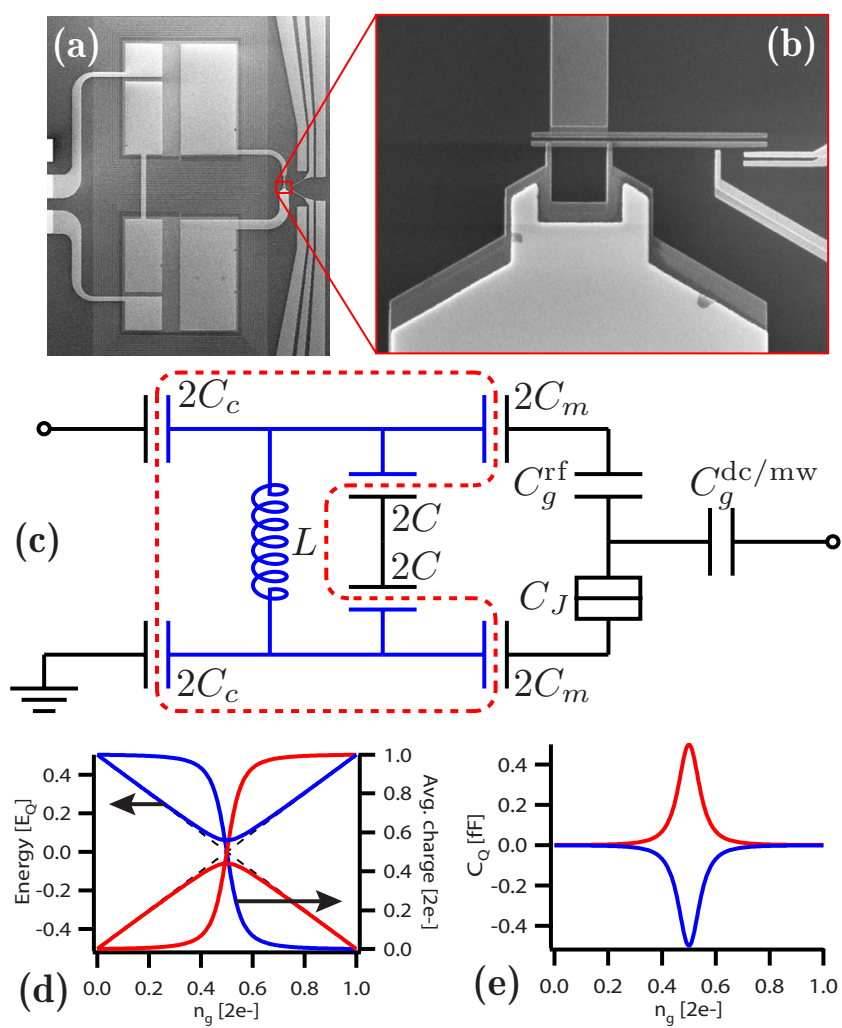

FIG. 1. (Color online) (a) A scanning electron micrograph of the resonator for sample A. The inductor can be seen spiraling around the plates of the capacitors with the upper part counter wound compared to the bottom part. (b) A scanning electron micrograph of the SCB for sample A. The SCB consists of a 5- $\mu$ m-long and 100-nmwide superconducting island. The SCB for sample B is the same except that the island is $8 \mu \mathrm{m}$ long in order to increase the gate capacitance. The island is connected to a reservoir through two small Josephson junctions in a SQUID geometry. The potential of the island is controlled by three different capacitive gates. One large rf gate, $C_{g}^{\mathrm{rf}}$ (on the top) is connected to the resonator and used for the readout. In addition, there is a dc gate, $C_{g}^{\mathrm{dc}}$, used to bias the SCB at the working point and a microwave gate, $C_{g}^{\mathrm{mw}}$, used for spectroscopy. (c) A schematic of the device. The parallel resonator consists of two metal layers separated by a thin insulating layer. The bottom layer is of $\mathrm{Nb}$ (within the dashed line), forming the inductor and bottom plates of the capacitors. Next is a $200 \mathrm{~nm}$ layer of silicon nitride (the dashed line) covering the whole sample and, finally, the top layer is $\mathrm{Al}$ (outside the dashed line) making the top plates of the capacitors as well as the SCB. (d) The energy of the two lowest energy eigenstates of the SCB, as well as the expectation value of the (excess) charge on the SCB island for each state as a function of the normalized gate voltage, $n_{g}$. (e) The quantum capacitance, $C_{Q}$, for the two eigenstates as a function of the normalized gate voltage, $n_{g}$.

\section{B. Quantum capacitance}

At the degeneracy point, the energy difference between the ground and excited state is, to first order, independent of the gate charge, $n_{g}$, which makes this the ideal bias point when the SCB is used as a qubit. At this bias point, the longest dephasing times are obtained. ${ }^{19}$ Therefore, this is often called the optimal point. If we want to detect the state of the SCB sitting at the optimal point, we cannot measure the charge, since the charges of the ground and exited state are the same at this point [see Fig. 1(d)]. Although the charges are the same for the two states, the derivatives of the charges with respect to the gate voltage differ and can be used for readout. We can define an effective capacitance of the SCB by calculating $C_{\text {eff }}=\partial\left\langle Q_{g}\right\rangle / \partial V_{g}$, where $\left\langle Q_{g}\right\rangle=C_{g} C_{J} V_{g} / C_{\Sigma}+2 e\langle n\rangle C_{g} / C_{\Sigma}$ is the average value of the injected charge on the gate capacitor. This effective capacitance, $C_{\text {eff }}=C_{\text {geom }}+C_{Q}$, will have two contributions, a geometric part $C_{\text {geom }}=C_{g} C_{J} /\left(C_{g}+C_{J}\right)$ consisting of the gate capacitance in series with the junction capacitance and a statedependent part that we call the quantum capacitance. ${ }^{13}$ This quantum capacitance, $C_{Q}$, takes the following form:

$$
C_{Q}^{g / e}= \pm \frac{C_{g}^{2}}{C_{\Sigma}} \frac{\alpha^{2}}{\left[\alpha^{2}+\left(1-2 n_{g}\right)^{2}\right]^{3 / 2}}= \pm \frac{C_{g}^{2}}{C_{\Sigma}} \frac{E_{Q} E_{J}^{2}}{\Delta E^{3}}
$$

for the ground (g) and first excited (e) state, where $\alpha=E_{J} / E_{Q}$ and $\Delta E=\sqrt{E_{J}^{2}+E_{Q}^{2}\left(1-2 n_{g}\right)^{2}}$ is the energy difference between the two states at a given $n_{g}$. $C_{Q}$ is equal in magnitude but has opposite signs for the ground and excited state, with $C_{Q}$ being negative for the excited state. In Fig. $1(\mathrm{e}), C_{Q}$ is plotted against the gate charge, $n_{g}$, for the ground and exited state of the SCB.

If we embed the SCB in a resonator [see Fig. 1(c)], the effect of the quantum capacitance will be to shift the resonance frequency. The reflection coefficient, $\Gamma=|\Gamma| \exp (\phi)$, of the resonator has a constant magnitude, i.e., $|\Gamma|=1$, since there are no dissipative elements in the resonator. The phase $\phi$, however has a sharp frequency dependence and close to the resonance frequency, $\omega_{0}=2 \pi f_{0}=1 / \sqrt{L\left(C+C_{c}\right)}$, the phase, $\phi$, can be approximated by the expression

$$
\phi=-\pi-2 \arctan \left(2 Q \frac{\omega-\omega_{0}}{\omega_{0}}\right),
$$

where $\omega=2 \pi f$ is the angular probe frequency and $Q=\left(C+C_{c}\right) / C_{c}^{2} Z_{0} \omega_{0}$ is the external quality factor of the resonator. Now, if the capacitance of the SCB is changed by $\Delta C_{Q}$, the phase of the rf signal at frequency $f_{0}$ reflected off the resonator will change by

$$
\Delta \phi \approx-2 \arctan \left(Q \frac{\Delta C_{Q}}{C+C_{c}}\right) .
$$

Here we have treated the resonator purely classically. A full quantum treatment, including the SCB, resonator, and transmission line can be found in the work of Johansson et al. ${ }^{20} \mathrm{It}$ is shown that this method of readout is quantum limited, ${ }^{20}$ meaning that no information is lost during the readout (no extra dephasing).

\section{Quasiparticles}

Quasiparticles are single-particle excitations of the superconducting condensate. Quasiparticle fluctuations have been studied as a source of noise in superconducting devices for some time. Thermodynamic fluctuations in the quasiparticle number, also know as generation-recombination noise, are an important source of noise at intermediate temperatures. ${ }^{21}$ 
Time-resolved measurements of these fluctuations have shown very good agreement between theory and experiment. ${ }^{22,23}$ At very low temperatures, were thermal quasiparticles should be suppressed, a significant population of quasiparticles is still observed in most experiments. The origin of these nonequilibrium quasiparticles is still unknown. However, it is clear that they remain an important source of noise.

The most significant source of quasiparticle noise in a SCB is commonly referred to as quasiparticle poisoning. When a nonequilibrium quasiparticle in the reservoir tunnels onto the SCB island, it shifts the potential of the island by $\pm e / C_{\Sigma}$. The random tunneling of quasiparticles on and off of the island therefore leads to large-amplitude telegraph noise in the island potential. Quasiparticle poisoning has been extensively studied, ${ }^{18,24,25}$ including the observation of individual tunneling events in real time. ${ }^{12,26}$ It has been shown that poisoning is well described by a simple kinetic model, starting from the assumption of a finite density of nonequilibrium quasiparticles in the leads. In this model, the tunneling rates are then dictated by the relative energies of the even (no quasiparticles on the island) and odd (one quasiparticle on the island) states. If the energy of the odd state is lower than the even state, a quasiparticle can be trapped on the island [see Fig. 3(a)]. The energy difference of the even and odd state is maximum at the charge degeneracy point, $n_{g}=0.5$, where it takes the value $\delta E=E_{Q} / 4-E_{J} / 2-\Delta_{i}+\Delta_{r}$. Here $\Delta_{i}$ and $\Delta_{r}$ are the superconducting energy gaps of the island and the reservoir, respectively. The average occupation of the two states is then simply determined by the Boltzmann factor $\exp \left(-\delta E / k_{B} T\right)$.

\section{DEVICE DESIGN AND FABRICATION}

When designing the readout circuit there is a trade off between having a good signal (large phase shift) and low backaction. The phase shift is roughly proportional to $Q C_{Q} /\left(C+C_{c}\right)$ [see Eq. (4)], meaning that for a fixed $Q$ value (i.e., measurement bandwidth) we want a small total capacitance in the resonator. However, the voltage noise of the environment will induce charge fluctuations on the SCB with a magnitude that scales with the same prefactor. Thus, by decreasing the total capacitance, $C+C_{c}$, you can increase the sensitivity (larger phase shift) at the cost of a larger backaction on the SCB.

The $Q$ value of the resonator sets the bandwidth, i.e., an upper limit on how fast we can measure. We designed the resonator to have an external $Q$ value of 100 which corresponds to a voltage time constant of $50 \mathrm{~ns}$ at $f_{0}=650 \mathrm{MHz}$. The internal $Q$ value is usually substantially larger and can therefore be ignored.

The devices were fabricated in a multilayer process. Starting from a high-resistivity silicon wafer with a native oxide, the wafer was first cleaned using rf back sputtering directly after which a 60 -nm-thick layer of niobium was sputtered. To pattern the niobium, we used a 20-nm-thick Al mask made by e-beam lithography and e-beam evaporation. The niobium was then etched in a $\mathrm{CF}_{4}$ plasma (with a small flow of oxygen) to form the inductor and bottom plates of the capacitors [see Fig. 1(c)]. The choice of niobium for the bottom layer made it possible to test the resonator in liquid helium (even with a top layer made of normal metal, e.g., gold). The Al mask was removed with a wet-etch solution based on phosphoric acid. Before depositing the insulator we cleaned the wafer in a $2 \% \mathrm{HF}$ solution for $30 \mathrm{~s}$ in order to remove most of the niobium oxide which has been found to degrade the $Q$ value of the niobium resonators. Using plasma-enhancedchemical-vapor deposition, we then deposited an insulating layer of $200 \mathrm{~nm}$ of silicon nitride. The silicon nitride layer covers the whole wafer; connections to the niobium layer are only made through capacitors. We chose silicon nitride since it is known to have low dielectric losses. ${ }^{27}$ After using a combination of DUV photolithography to define bonding pads along with e-beam lithography to define quasiparticle traps, ${ }^{28}$ a 3/80/10-nm-thick layer of Ti/Au/Pd was deposited by e-beam evaporation. Finally, the layer containing the SCB was made by e-beam lithography and two-angle shadow evaporation of $10+30 \mathrm{~nm}$ of aluminum with 6 min of oxidation at 4 mbar. The thickness of the island $(10 \mathrm{~nm})$ was chosen to be much thinner than for the reservoir $(30 \mathrm{~nm})$ in order to enhance the superconducting gap, $\Delta_{i}>\Delta_{r}$, of the island compared to the reservoir. ${ }^{29}$ This was done to reduce quasiparticle poisoning. ${ }^{24,30,31}$

\section{MEASUREMENTS}

\section{A. Measurement setup}

The devices were cooled in a dilution refrigerator with a base temperature of about $20 \mathrm{mK}$. For the readout, we used an Aeroflex 3020 signal generator to produce the rf signal. The signal was heavily attenuated and filtered and was fed to the resonator via a Pamtech circulator positioned at the mixing chamber. The reflected signal was amplified by a Quinstar amplifier at $4 \mathrm{~K}$ with a nominal noise temperature of $1 \mathrm{~K}$. The in-phase and quadrature components of the signal were finally measured using an Aeroflex 3030 vector digitizer. A schematic of the measurement setup can be found in Fig. 2.

\section{B. Device characterization}

In the following sections, we will show measurements of two different devices, referred to as sample A and sample B. In order to characterize the devices and extract parameters, the resonator was first measured using a network analyzer. From the measured phase response, we extracted the resonance frequency, $f_{0}$, and the $Q$ value of the resonator. We extracted both the Cooper-pair charging energy, $E_{Q}$, and the maximum Josephson energy, $E_{J}^{\max }$ by conventional spectroscopy, while applying a perpendicular magnetic field through the SQUID loop of the SCB, and thereby tuning $E_{J}$. Finally, by measuring $C_{Q}$ as a function of gate charge, we extracted the rf gate capacitance and the total resonator capacitance. From the expression for the $Q$ value and the resonance frequency, we can then extract the parameters for the individual components of the resonator. The extracted parameters for the two devices are presented in Table I. The values of the parameters for sample B correspond reasonably well to the 




FIG. 2. The measurement setup used in the experiment. The sample (inside the dashed line) is mounted inside a copper box on the mixing chamber of a dilution refrigerator, which reaches a base temperature of $20 \mathrm{mK}$. The readout pulse is created using a Aeroflex 3020 signal generator (with built in IQ modulation). It is heavily attenuated and coupled through a circulator located at the mixing chamber and filtered through two RLC waveguide filters before reaching the resonator. On the way up, the signal is first amplified at $4.2 \mathrm{~K}$ with a Quinstar amplifier with a noise temperature of $1 \mathrm{~K}$ and then amplified again at room temperature with a Mini-Circuits amplifier. Before the signal is finally digitized using an Aeroflex 3030 vector analyzer (with a $33 \mathrm{MHz}$ bandwidth), it is first filtered using an RLC bandpass filter to reject image noise. Gray rectangles represent $50 \Omega$ attenuators with the attenuation indicated in decibel whereas the rectangle labeled 1:101 is a voltage divider with that ratio. Filters are labeled as follows: $\mathrm{LP}=$ low-pass filter, $\mathrm{HP}=$ high-pass filter, $\mathrm{BP}=$ bandpass filter, and $\mathrm{PF}=$ powder filter.

geometrically identical device that was used in Ref. 32. The gate capacitance for sample A roughly agrees with what you would expect from the $5 \mu \mathrm{m}$ island compared to the capacitance of the $8 \mu \mathrm{m}$ island in sample B. If we the insert the parameters from Table I into Eq. (4), we calculate an expected phase shift of $11^{\circ}$ for sample A and $30^{\circ}$ for sample B between the two parity states when biased at the degeneracy point.

\section{Readout performance}

We have fabricated and measured a number of samples. All devices we have measured so far have been poisoned, meaning that quasiparticles cause switching between the two parity states of having an even or odd number of quasiparticles on the island. The switching between these parities happens on the time scale of a few microseconds. Although this
TABLE I. The extracted parameters for the two samples.

\begin{tabular}{lcc}
\hline \hline & Sample A & Sample B \\
\hline$f_{0}(\mathrm{MHz})$ & 676 & 663 \\
$Q$ & 128 & 130 \\
$L(\mathrm{nH})$ & 151 & 324 \\
$C(\mathrm{fF})$ & 251 & 97 \\
$C_{c}(\mathrm{fF})$ & 116 & 81 \\
$C_{g}^{\mathrm{rf}}(\mathrm{fF})$ & 0.2 & 0.3 \\
$E_{Q}(\mathrm{GHz})$ & 62 & 48 \\
$E_{J}^{\max }(\mathrm{GHz})$ & 7.2 & 7.4 \\
\hline \hline
\end{tabular}

is far from ideal for many applications, it has given us a way to characterize the readout. When a quasiparticle tunnels on or off the island the quantum capacitance of the SCB will change, and can thus be detected as a change in the phase of the reflected signal from the resonator. The measured timeaveraged phase for sample B is shown in Fig. 3(b) as function of $n_{g}$. In Fig. 3(c), we show the time dependence of the phase measured at the degeneracy point, $n_{g}=0.5$. Most of the time the SCB is in the odd state with a phase shift of about $0^{\circ}$. However, now and then the extra quasiparticle escapes the island and the SCB spends short periods in the even state with a phase shift close to $-30^{\circ}$.

While sitting at the degeneracy point, $n_{g}=0.5$, we have also performed pulsed measurements of the state. We send down a Gaussian pulse, with a length defined as the full width at half maximum (FWHM), and measure the phase of the reflected pulse. In order to optimize the signal to noise of the measured response, we used a so-called matched filter, where the time traces of the measured in-phase and quadrature component $\left(V_{I}\right.$ and $\left.V_{Q}\right)$ are multiplied with a Gaussian template (with the same shape as the one generated by the signal generator). The product is then integrated and a single value is extracted. This is done for both $V_{I}$ and $V_{Q}$ and we can then calculate the phase $\phi=\arctan \left(V_{Q} / V_{I}\right)$. We perform $10^{5}$ of these measurements and make histograms of the measured phase. We typically see two peaks centered at different phases corresponding to the two parity states. We fit a double Gaussian (both with the same standard deviation) to the histograms [see Figs. 4(a) and 4(b)]. We define the signal-tonoise ratio (SNR) of the measurement as the peak separation divided by the standard deviation. This is performed for different pulse lengths and we extract the SNR as a function of the measurement time, Figs. 4(c) and 4(d). The SNR roughly follows the expected square-root dependence on the measurement time. To reach a SNR $\gtrsim 1$ we need a pulse length on the order of 50-100 ns in both samples. The shortest measurement time was here limited by the time constant of resonator which was about $60 \mathrm{~ns}$ for both of the samples.

\section{Quasiparticle relaxation and state preparation}

We know that by sitting at the even degeneracy point the system will eventually relax into the odd parity state given that $E_{Q} / 4-E_{J} / 2>\Delta_{i}-\Delta_{l}$ (see Fig. 3). We wanted to study how fast this process is and to what extent you can prepare 

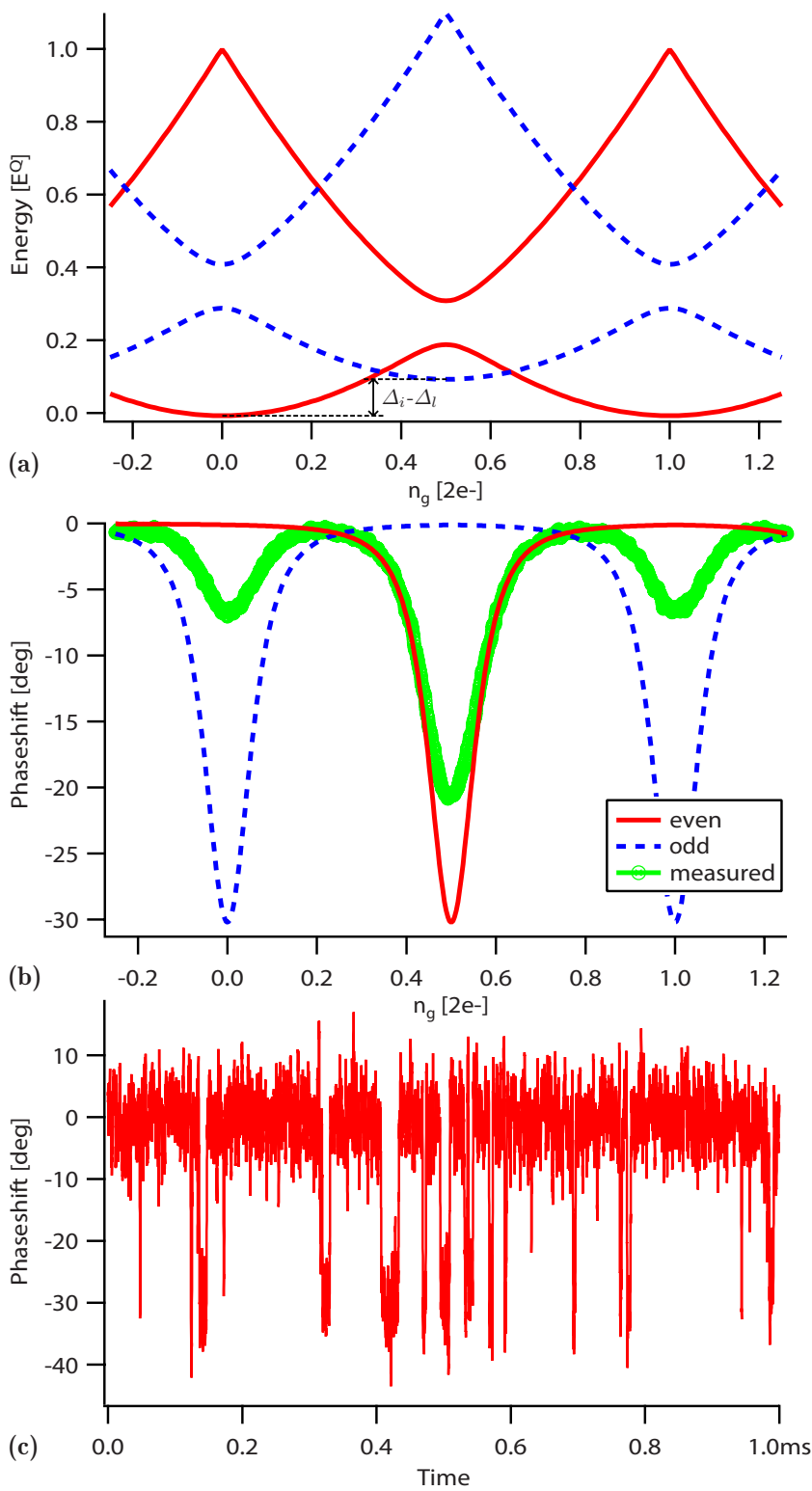

FIG. 3. (Color online) (a) The energy as a function of $n_{g}$ for the even (solid) and odd (dashed) parity of the SCB island. The odd state energy is shifted by the difference in the superconducting gaps of the island and the leads, $\Delta_{i}-\Delta_{l}$. If $E_{Q} / 4-E_{J} / 2>\Delta_{i}-\Delta_{l}$ the island will form a trap for quasiparticles at $n_{g}=0.5$. (The value of $\Delta_{i}-\Delta_{l}$ in the figure does not represent the real value of the system and the figure is only meant as an illustration.) (b) The phase shift as a function of the gate charge, $n_{g}$, for the even (solid) and odd (dashed) state. We also show the average phase for sample B, which shows contributions from both parity states, measured while repetitively sweeping the dc gate at a repetition rate of $5 \mathrm{kHz}$. The rate of the dc sweep was comparable to the quasiparticle tunneling rate, such that the quasiparticle tunneling probability during one period was low. The SCB then spent most of the time in the even state, which has, on average, a lower energy than the odd state. (c) A typical time trace of the measured phase, from a device identical to sample B, when sitting at the even degeneracy point, $n_{g}=0.5$. The SCB spends most of the time in the odd state $\left(\phi=0^{\circ}\right)$ but makes short excursions to the even state $\left(\phi=-30^{\circ}\right)$.


FIG. 4. (Color online) Sitting at $n_{g}=0.5$, we perform pulsed measurements and extract the phase of each pulse. For three different pulse lengths, we repeat the measurements many times and make histograms of the phases (bars). In (a), we show histograms for measurements on sample A and in (b) for sample B. Sample A was designed to have a larger total capacitance for the resonator and a smaller rf-gate capacitance than in sample B. This reduces the backaction of the measurement at the expense of lowering the SNR. To the histograms, we fit a double Gaussian (solid line) with the same standard deviation for the two peaks. We then define the SNR of the measurement as the peak separation divided by the standard deviation. The above procedure is repeated for different pulse lengths, ranging from $20 \mathrm{~ns}$ to $1 \mu \mathrm{s}$. The SNR is then plotted as function of the pulse length for sample A in (c) and for sample B in (d). We see that the SNR roughly follows the expected square-root dependence on the measurement time. Since sample A was designed to have a lower backaction, we expect it to have a lower signal. The calculated phase shift is $11^{\circ}$ for sample A and $30^{\circ}$ for sample B. This value is however calculated for very low probe powers. In these measurements, the phase shift is reduced by the relatively large measurement power.

the SCB in a certain parity state. We start by letting the system equilibrate biased at the odd degeneracy point, i.e., $n_{g}=0$, thereby preparing the even state. We then pulse the gate to the even degeneracy point (at $t=100 \mu \mathrm{s}$ ) and observe the dynamics. From a long-time trace, including 1000 pulses, we divide each repetition into $0.5 \mu$ s increments. We extract the average phase from each increment and each repetition. We then make a histogram of the phase for each increment as a function of time [see Fig. 5(b)]. We fit the histograms to a double Gaussian and extract the occupation probability of the even and odd state as a function of time [see Fig. 5(c)]. 

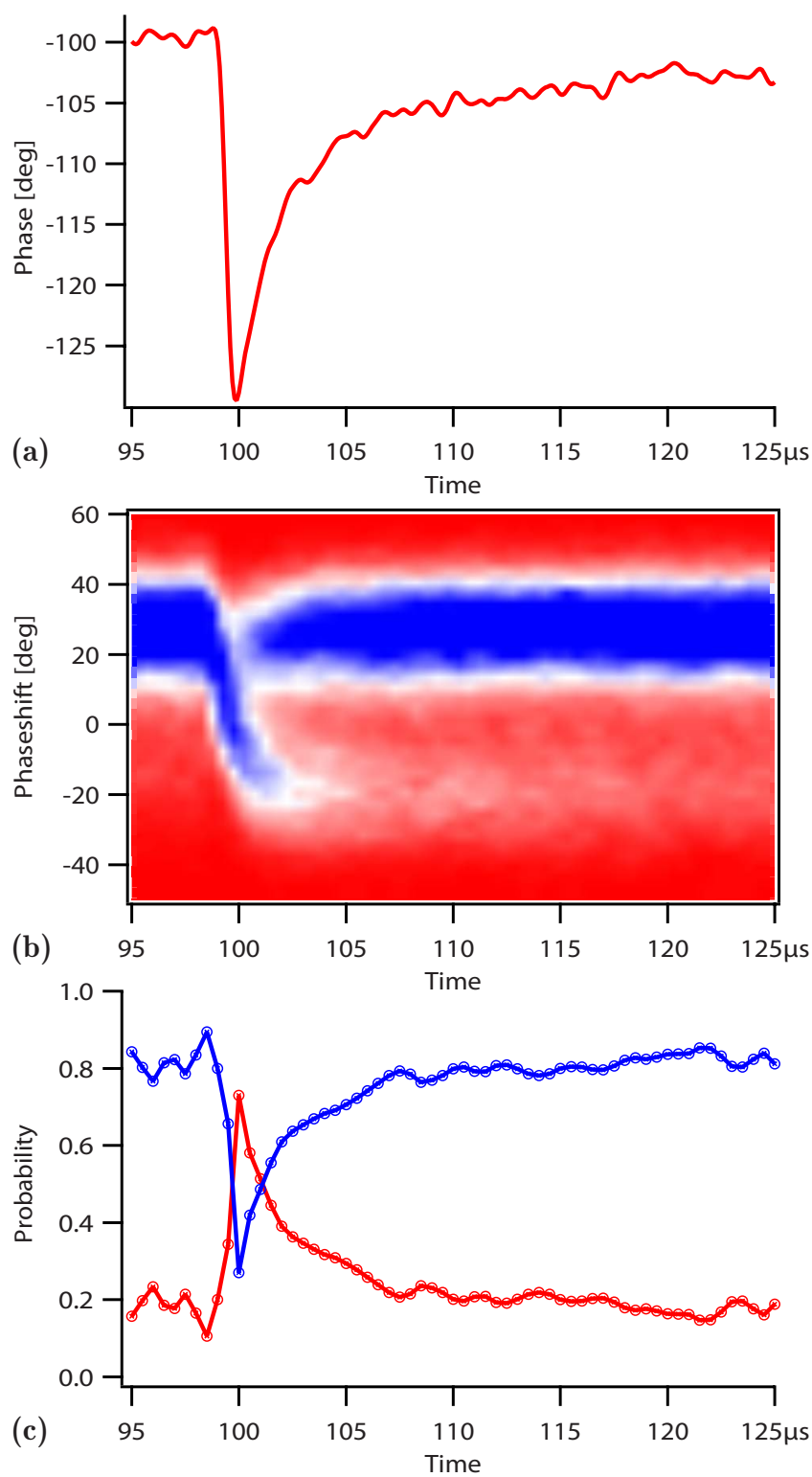

FIG. 5. (Color online) Measurement of the quasiparticle relaxation in sample B. (a) The average of 1000 traces of the measured phase after pulsing the gate from the odd degeneracy point $\left(n_{g}=0\right)$ (at time $\left.100 \mu \mathrm{s}\right)$ to the even degeneracy point $\left(n_{g}=0.5\right)$ and continuously monitoring the phase as a function of time. (b) Histograms of the measured state as a function of time. The measured phase for each repetition is divided into $0.5 \mu$ s increments. We then make a histogram of the average phase for each increment, measured from the start of the pulse. This produces a histogram as a function of time. (c) Occupation probability of the even (lower) and odd (upper) state as function of time extracted from the histograms in (b). After pulsing to the even degeneracy point, the probabilities are inverted suggesting that we should be able to prepare the system in the even state with high probability even if the device has significant quasiparticle poisoning.
Sitting at the odd degeneracy point, $n_{g}=0$, there is an equilibrium probability of more than $80 \%$ of being in the even state and when we pulse to the even degeneracy point most of the probability is preserved. Eventually the system equilibrates and then the probability is reduced to $\sim 20 \%$. From the relaxation of the probabilities, we extract an equilibration time of $2.8 \mu \mathrm{s}$. As a comparison, we show the average time trace [see Fig. 5(a)] where we have taken the average of the full time traces from different pulses. From this, we extract a relaxation time of $3 \mu \mathrm{s}$, which is in good agreement. Since the quasiparticle relaxation time is relatively long it suggests that, even if the device is poisoned, we should be able to prepare the box in the even state with high probability and perform useful measurements. For example, in a qubit experiment where poisoning is unwanted, one could prepare the parity state of the system before performing qubit gates and readout, thereby increasing the fidelity of the measurements. The observed parity relaxation time of $\sim 3 \mu$ s is significantly longer than the observed dephasing time of charge qubits and would therefore not limit coherent operation. Parity state preparation could also be useful when the SCB is used as an electrometer as, to maximize the sensitivity, one has to bias the SCB near the even degeneracy point before performing the measurement.

\section{CONCLUSIONS}

We have fabricated and tested two samples with a SCB together with an on-chip lumped element resonator. The resonators were optimized for readout speed, with a $Q$ value around 100 . We have characterized the readout by employing the effect of quasiparticle poisoning and found that for readout pulses of length 50-100 ns we get a SNR greater then 1 .

\section{ACKNOWLEDGMENTS}

We thank the members of the Quantum Device Physics and Applied Quantum Physics groups for useful discussions. Special thanks goes to Alexey Pavolotsky for providing the $\mathrm{Nb}$ films. The samples were made at the nanofabrication laboratory at Chalmers. The work was supported by the Swedish VR and SSF, the Wallenberg foundation, the EU under the projects EuroSQIP and SCOPE. This research was also funded by the Office of the Director of National Intelligence (ODNI), Intelligence Advanced Research Projects Activity (IARPA), through the Army Research Office. All statements of fact, opinion or conclusions contained herein are those of the authors and should not be construed as representing the official views or policies of IARPA, the ODNI, or the U.S. Government. 
*fredrik.persson@chalmers.se

†per.delsing@chalmers.se

${ }^{1}$ Y. Makhlin, G. Schön, and A. Shnirman, Rev. Mod. Phys. 73, 357 (2001).

${ }^{2}$ J. H. Plantenberg, P. C. de Groot, C. J. P. M. Harmans, and J. E. Mooij, Nature (London) 447, 836 (2007).

${ }^{3}$ R. McDermott, R. W. Simmonds, M. Steffen, K. B. Cooper, K. Cicak, K. D. Osborn, S. Oh, D. P. Pappas, and J. M. Martinis, Science 307, 1299 (2005).

${ }^{4}$ J. Koch, T. M. Yu, J. Gambetta, A. A. Houck, D. I. Schuster, J. Majer, A. Blais, M. H. Devoret, S. M. Girvin, and R. J. Schoelkopf, Phys. Rev. A 76, 042319 (2007).

${ }^{5}$ T. A. Fulton and G. J. Dolan, Phys. Rev. Lett. 59, 109 (1987).

${ }^{6}$ R. J. Schoelkopf, P. Wahlgren, A. A. Kozhevnikov, P. Delsing, and D. E. Prober, Science 280, 1238 (1998).

${ }^{7}$ H. Brenning, S. Kafanov, T. Duty, S. Kubatkin, and P. Delsing, J. Appl. Phys. 100, 114321 (2006).

${ }^{8}$ M. Büttiker, Phys. Rev. B 36, 3548 (1987).

${ }^{9}$ V. Bouchiat, D. Vion, P. Joyez, D. Esteve, and M. H. Devoret, Phys. Scr. T T76, 165 (1998).

${ }^{10}$ Y. Nakamura, Y. A. Pashkin, and J. S. Tsai, Nature (London) 398, 786 (1999).

${ }^{11}$ M. D. Shaw, J. Bueno, P. Day, C. M. Bradford, and P. M. Echternach, Phys. Rev. B 79, 144511 (2009).

${ }^{12}$ A. J. Ferguson, N. A. Court, F. E. Hudson, and R. G. Clark, Phys. Rev. Lett. 97, 106603 (2006).

${ }^{13}$ T. Duty, G. Johansson, K. Bladh, D. Gunnarsson, C. Wilson, and P. Delsing, Phys. Rev. Lett. 95, 206807 (2005).

${ }^{14}$ M. A. Sillanpää, T. Lehtinen, A. Paila, Y. Makhlin, L. Roschier, and P. J. Hakonen, Phys. Rev. Lett. 95, 206806 (2005).

${ }^{15}$ C. M. Wilson, T. Duty, F. Persson, M. Sandberg, G. Johansson, and P. Delsing, Phys. Rev. Lett. 98, 257003 (2007).

${ }^{16}$ C. M. Wilson, G. Johansson, T. Duty, F. Persson, M. Sandberg, and P. Delsing, Phys. Rev. B 81, 024520 (2010).

${ }^{17}$ M. D. Shaw, J. F. Schneiderman, J. Bueno, B. S. Palmer, P. Delsing, and P. M. Echternach, Phys. Rev. B 79, 014516 (2009).

${ }^{18}$ M. D. Shaw, R. M. Lutchyn, P. Delsing, and P. M. Echternach, Phys. Rev. B 78, 024503 (2008).

${ }^{19}$ D. Vion, A. Aassime, A. Cottet, P. Joyez, H. Pothier, C. Urbina, D. Esteve, and M. H. Devoret, Science 296, 886 (2002).

${ }^{20} \mathrm{G}$. Johansson, L. Tornberg, and C. M. Wilson, Phys. Rev. B 74, 100504(R) (2006).

${ }^{21}$ C. M. Wilson, L. Frunzio, K. Segall, L. Li, D. E. Prober, D. Schiminovich, B. Mazin, C. Martin, and R. Vasquez, IEEE Trans. Appl. Supercond. 11, 645 (2001).

${ }^{22}$ C. M. Wilson and D. E. Prober, Phys. Rev. B 69, 094524 (2004).

${ }^{23}$ C. M. Wilson, L. Frunzio, and D. E. Prober, Phys. Rev. Lett. 87, 067004 (2001).

${ }^{24}$ J. Aumentado, M. W. Keller, J. M. Martinis, and M. H. Devoret, Phys. Rev. Lett. 92, 066802 (2004).

${ }^{25}$ R. M. Lutchyn and L. I. Glazman, Phys. Rev. B 75, 184520 (2007).

${ }^{26}$ O. Naaman and J. Aumentado, Phys. Rev. B 73, 172504 (2006).

${ }^{27}$ J. M. Martinis et al., Phys. Rev. Lett. 95, 210503 (2005).

${ }^{28}$ M. J. Rooks, S. Wind, P. McEuen, and D. E. Prober, J. Vac. Sci. Technol. B 5, 318 (1987).

${ }^{29}$ R. Meservey and P. M. Tedrow, J. Appl. Phys. 42, 51 (1971).

${ }^{30}$ D. Gunnarsson, T. Duty, K. Bladh, and P. Delsing, Phys. Rev. B 70, 224523 (2004).

${ }^{31}$ N. A. Court, Ph.D. thesis, The University of South Wales, 2008.

${ }^{32}$ F. Persson, C. M. Wilson, M. Sandberg, G. Johansson, and P. Delsing, Nano Lett. 10, 953 (2010). 